\title{
Error-Power Tradeoffs in QCA Design
}

\author{
Saket Srivastava*, Sudeep Sarkar ${ }^{\dagger}$ and Sanjukta Bhanja* \\ ${ }^{*}$ Electrical Engineering, ${ }^{\dagger}$ Computer Science and Engineering \\ University of South Florida, Tampa, Florida. \\ Emails: ssrivast@mail.usf.edu, sarkar@cse.usf.edu, bhanja@eng.usf.edu
}

\begin{abstract}
In this work we present an error-power tradeoff study in a Quantum-dot Cellular Automata (QCA) circuit design. Device parameter variation to optimize performance is a very crucial step in the development of a technology. In this work we vary the maximum kink energy of a QCA circuit to perform an error-power tradeoff study in QCA design. We make use of graphical probabilistic models to estimate polarization errors and non-adiabatic energy dissipated in a clocked QCA circuit and demonstrate the tradeoff studies on the basic QCA circuits such as majority gate and inverter. We also show how this study can be used by comparing two single bit adder designs. The study will be of great use to designers and fabrication scientists to choose the most optimum size and spacing of QCA cells to fabricate QCA logic designs.
\end{abstract}

\section{INTRODUCTION}

Quantum-dot Cellular Automata (QCA) is one of the most promising technologies being currently researched. It uses an interesting charge coupled computing paradigm to perform computation [1]. A lot of progress has been made recently to fabricate QCA using magnetic dots [2], [3] and molecules [4], [5] that will enable it to perform room temperature operations.

While there have been experimental studies related to defect and fault tolerance in QCA [6], [7], [8], not much work has been done to study the effects of variation in device parameters on error and power in QCA design. Similar studies have been the hallmark of CMOS research over the years that contributed significantly in the development of CMOS technology. It is natural to perform such a study with respect to parameter variations in QCA. We perform a study of error and power dissipation in a clocked QCA design by varying one of the most crucial parameter in QCA design; the kink energy. Kink energy is the energy cost of keeping two adjacent cells in opposite polarization and varies with the size of a QCA cell and the grid spacing. We analyze the effects of kink energy with a design perspective to help designers and fabrication scientists to choose the most optimum size of QCA cell and spacing between adjacent QCA cells.

In [9] it has been shown how to calculate the ground state polarization probabilities and build a graphical probabilistic model based on that. We used these graphical probabilistic models to detemine thermal error at the output at different temperatures. In [10], an efficient method, based on graphical probabilistic models was presented, to compute the N-lowest energy modes of a clocked QCA circuit. In QCA, an erroneous state may result due to the failure of the clocking scheme to switch portions of the circuit to its new ground state with change in input. This error state of a single cell in turn causes the error in the neighboring cells resulting in an erroneous
TABLE I

DIFFERENT TYPES OF QCA CELLS AND GRID SPACING USED IN THIS STUDY

\begin{tabular}{||l|l|l|l||}
\hline QCA cell & Size & Grid Spacing & Associated Kink Energy \\
\hline \hline Cell-1 & $10 \mathrm{~nm}$ & $5 \mathrm{~nm}$ & $E_{k 1}=4 E_{k}$ \\
\hline Cell-2 & $20 \mathrm{~nm}$ & $10 \mathrm{~nm}$ & $E_{k 2}=2 E_{k}$ \\
\hline Cell-3 & $40 \mathrm{~nm}$ & $20 \mathrm{~nm}$ & $E_{k 3}=E_{k}$ \\
\hline
\end{tabular}

output. Due to the quantum mechanical nature of operation of a QCA device, temperature plays an important role in determining the ground state polarization of each cell. Power dissipation in a QCA circuit primarily results due the the application of a non-adiabatic clocking scheme. We have also seen in [11], how clock energy affects the overall power dissipation in a QCA circuit.

In this work we perform studies to determine the error and power tradeoff in a QCA circuit design by studying the effect of kink energy on the output error and power dissipation in a QCA circuit. We use three different sizes of QCA cells and grid spacing to study the polarization and power dissipation for basic QCA circuits using these cells. The three different types of cell sizes used in this study are elaborated in Table I. Here $E_{k 1}$ is the maximum kink energy for the cell layout with smallest cell dimensions (and grid spacing). Similarly, $E_{k 3}$ is the maximum kink energy for the QCA layout with largest cell dimensions (and grid spacing). It can be seen from Table I, $E_{k 1}=2 E_{k 2}=4 E_{k 3}$.

We first simulate a number of basic QCA circuits such as majority gate and inverter to study the polarization error at the output for each input vector set. We also determine the power dissipation in these circuits for different kink energies. All other parameters such as temperature and clock energy are kept constant. We show how this study can be used by comparing two single bit adder designs. The study will be of great use to designers and fabrication scientists to choose the most optimum size and spacing of QCA cells to fabricate QCA logic designs.

\section{KINK ENERGY}

Two electrons in a simple four dot QCA cell occupy diagonally opposite dots in the cell due to mutual repulsion of like charges. A QCA cell can be in any one of the two possible states depending on the polarization of charges in the cell. The two polarized states are represented as $\mathrm{P}=+1$ and 


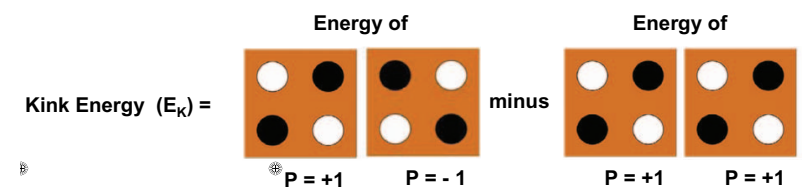

Fig. 1. Kink energy between two neighboring QCA cells

$P=-1$. Electrostatic interaction between charges in two QCA Cells is given as:

$$
E^{m}=\frac{1}{4 \pi \varepsilon_{o} \varepsilon_{r}} \sum_{i=1}^{4} \sum_{j=1}^{4} \frac{q_{i}^{m} q_{j}^{k}}{\left|r_{i}{ }^{m}-r_{j}^{k}\right|}
$$

This interaction determines the kink energy between two cells.

$$
E_{\text {kink }}=E_{\text {opp.polarization }}-E_{\text {samepolarization }}
$$

Kink energy (Fig 1) is the energy cost of two neighboring QCA cells having opposite polarization. Kink energy between two cells depends on the dimension of the QCA cell as well as the spacing between adjacent cells. It does not depend on the temperature.

\section{ESTIMATION OF ERROR}

A QCA system can be erroneous if it fails to settle down to ground state. In order to estimate error in a QCA circuit, we use the probabilistic error model presented in [10]. This model arrives at the probability of error in a QCA system using ground state polarization probabilities and using it to estimate error at the output of a QCA circuit represented by a graphical probabilistic network. A brief description of this model is provided below.

The steady state polarization of a QCA cell can be derived from the Hamiltonian of the cell using Hartree approximation. Expression of Hamiltonian is shown in Eq. 3 [12].

$$
\mathbf{H}=\left[\begin{array}{cc}
-\frac{1}{2} \sum_{i} E_{k} P_{i} f_{i} & -\gamma \\
-\gamma & \frac{1}{2} \sum_{i} E_{k} P_{i} f_{i}
\end{array}\right]=\left[\begin{array}{cc}
-\frac{1}{2} E_{k} \bar{P} & -\gamma \\
-\gamma & \frac{1}{2} E_{k} \bar{P}
\end{array}\right]
$$

where the sums are over the cells in the local neighborhood. $E_{k}$ is the "kink energy" . $f_{i}$ is the geometric factor capturing electrostatic fall off with distance between cells. $P_{i}$ is the polarization of the $i$-th cell. And, $\gamma$ is the tunneling energy between two cell states, which is controlled by the clocking mechanism. The notation can be further simplified by using $\bar{P}$ to denote the weighted sum of the neighborhood polarizations $\sum_{i} P_{i} f_{i}$. Using this Hamiltonian the steady state polarization is given by

$P^{s s}=-\lambda_{\mathbf{3}}^{s s}=\rho_{11}^{s s}-\rho_{00}^{s s}=\frac{E_{k} \bar{P}}{\sqrt{E_{k}^{2} \bar{P}^{2}+4 \gamma^{2}}} \tanh \left(\frac{\sqrt{E_{k}^{2} \bar{P}^{2} / 4+\gamma^{2}}}{k T}\right)$

Eq. 4 can be written as

$$
P^{s s}=\frac{E}{\Omega} \tanh (\Delta)
$$

where $E=0.5 \sum_{i} E_{k} P_{i} f_{i}$, total kink energy and Rabi frequency $\Omega=\sqrt{E_{k}^{2} \bar{P}^{2} / 4+\gamma^{2}}$ and $\Delta=\frac{\Omega}{k T}$ is the thermal ratio. We will use the above equation to arrive at the probabilities of observing (upon making a measurement) the system in each of the two states. Specifically, $\rho_{11}^{s s}=0.5\left(1+P^{s s}\right)$ and $\rho_{00}^{s s}=$ $0.5\left(1-P^{s s}\right)$, where we made use of the fact that $\rho_{00}^{s s}+\rho_{11}^{s s}=1$. Where $\rho_{11}^{s s}\left(\rho_{00}^{s s}\right)$ is the probability of observing the system (here a QCA cell) in state 1 (state 0).

The value of steady state polarization proabability is used to estimate error in a QCA circuit using graphical probabilistic model [10].

\section{Estimation of POWER DisSIPATION}

We make use of the non-adiabatic power dissipation model presented in [11], to estimate switching power losses is a QCA circuit. This model was derived from the the quasi-adiabatic model presented by Timler et.al. [13]. According to this model, the equation for the instantaneous power is given by:

$$
P_{\text {total }}=\frac{d}{d t} E=\frac{\hbar}{2}\left(\frac{d}{d t} \vec{\Gamma}\right) \cdot \vec{\lambda}+\frac{\hbar}{2} \vec{\Gamma} \cdot\left(\frac{d}{d t} \vec{\lambda}\right)
$$

Where $\vec{\lambda}$ the coherence vector and $\vec{\Gamma}$ is the three-dimensional energy vector. The first term captures the power in and out of the clock and cell to cell power flow. We are concerned with $P_{\text {diss }}$ which represents the instantaneous dissipated power. Power dissipated during switching can thus be calculated by integrating $P_{\text {diss }}$ over time.

$$
P_{\text {diss }}(t)=\frac{\hbar}{2} \vec{\Gamma}(t) \cdot\left(\frac{d}{d t} \vec{\lambda}(t)\right)
$$

The energy vector $\vec{\Gamma}$ of a QCA cell varies with kink energy thereby affecting the overall power dissipation . Please see [13] for more details about these terms.

\section{RESUlts}

In this section we present the results obtained from the study of variation of kink energy on error and power dissipated in the circuits. We obtained these results by simulating each of the circuits at a constant temperature of $2 \mathrm{~K}$. We used QCADesigner [14] tool to design QCA circuits and then analyzed it using our graphical probabilistic modeling scheme.

\section{A. Output Node Polarization Error}

We quantify the error in a circuit as a measure of its output node polarization. In prior work [9], [15], using temperature as a variable and keeping the kink energy constant, it has been shown how the output node polarization drops steadily with rise in temperature leading to more erroneous outputs. This effect becomes more and more significant with the increase in the number of cells in a design. Hence two different designs representing similar logic but having unequal number of cells will have different polarizations at the output nodes.

Similarly, in this study, by varying the kink energy of the circuit and keeping the temperature constant we see that the gain (drop) in output node polarization of a circuit is directly proportional to the increase (decrease) in maximum 


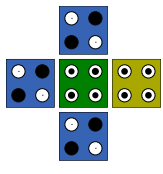

(a) (b)

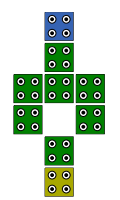

Fig. 2. (a) QCA Majority Gate (b) Inverter

TABLE II

OUTPUT NODE POLARIZATION OF A SIMPLE MAJORITY GATE FOR DIFFERENT KINK ENERGIES

\begin{tabular}{|c|c|c|c|}
\hline & \multicolumn{3}{|c|}{ Maximum Kink Energy $\left(E_{k}\right)$} \\
\hline Input & $\begin{array}{l}E_{k 3}=0.75 \\
\mathrm{meV}\end{array}$ & $E_{k 2}=1.5 \mathrm{meV}$ & $E_{k 1}=3.0 \mathrm{meV}$ \\
\hline $\begin{array}{lllllll} & 0 & 0\end{array}$ & 0.9278 & 0.9999 & 1.0000 \\
\hline $\begin{array}{lll}0 & 0 & 1\end{array}$ & 0.9880 & 0.9999 & 1.0000 \\
\hline $\begin{array}{llll}0 & 1 & 0\end{array}$ & 0.9880 & 0.9999 & 1.0000 \\
\hline $\begin{array}{llll}0 & 1 & 1\end{array}$ & 0.9075 & 0.9999 & 1.0000 \\
\hline 100 & 0.9075 & 0.9999 & 1.0000 \\
\hline $\begin{array}{lll}10 & 1\end{array}$ & 0.9880 & 0.9999 & 1.0000 \\
\hline 110 & 0.9880 & 0.9999 & 1.0000 \\
\hline 111 & 0.9278 & 0.9999 & 1.0000 \\
\hline
\end{tabular}

kink energy $\left(E_{k}\right)$ of the circuit. Here increase in $E_{k}$ refers to decrease in QCA cell size and grid spacing. Similar effect was seen for different values of temperature.

As an example, refer to the output node polarization of a simple majority gate (Fig. 2) shown in Table II. As we have shown earlier, we first form a Bayesian network of the QCA circuit and use a graphical simulator to obtain the polarization probability for each QCA cell (represented as a node) in the design. We can see that the polarization probability at the output of the Bayesian network rises with the increase in kink energy. Hence, we can infer that designs with lower value of maximum kink energy are more prone to error and this error is more significant when the number of cells in a design increases or the temperature is raised. Table III shows the output node polarization probability of a QCA inverter.

TABLE III

OUTPUT NODE POLARIZATION OF A QCA INVERTER FOR DIFFERENT KINK ENERGIES

\begin{tabular}{||l||l|l|l||}
\hline \multicolumn{1}{||c||}{} & \multicolumn{3}{c||}{ Maximum Kink Energy $\left(E_{k}\right)$} \\
\hline Input & $\begin{array}{l}E_{k 3}=0.75 \\
\mathrm{meV}\end{array}$ & $E_{k 2}=1.5 \mathrm{meV}$ & $E_{k 1}=3.0 \mathrm{meV}$ \\
\hline \hline 0 & 0.9750 & 0.9998 & 1.0000 \\
\hline 1 & 0.9843 & 0.9998 & 1.0000 \\
\hline
\end{tabular}

TABLE IV

POWER DISSIPATION IN QCA MAJORITY GATE FOR DIFFERENT KINK ENERGIES

\begin{tabular}{||l||l|l|l||}
\hline \multicolumn{1}{||c||}{} & \multicolumn{3}{c||}{ Maximum Kink Energy $\left(E_{k}\right)$} \\
\hline & $\begin{array}{l}E_{k 3}=0.75 \\
\mathrm{meV}\end{array}$ & $E_{k 2}=1.5 \mathrm{meV}$ & $E_{k 1}=3.0 \mathrm{meV}$ \\
\hline \hline Max $E_{\text {diss }}(\mathrm{meV})$ & 0.0051 & 0.0147 & 0.0294 \\
\hline Avg $E_{\text {diss }}(\mathrm{meV})$ & 0.0018 & 0.0060 & 0.0120 \\
\hline Min $E_{\text {diss }}(\mathrm{meV})$ & 0.0002 & 0.0008 & 0.0015 \\
\hline Avg $E_{\text {leak }}(\mathrm{meV})$ & 0.0003 & 0.0009 & 0.0018 \\
\hline Avg $E_{\text {sw }}(\mathrm{meV})$ & 0.0015 & 0.0051 & 0.0102 \\
\hline
\end{tabular}

TABLE V

POWER DISSIPATION IN QCA INVERTER FOR DIFFERENT KINK ENERGIES

\begin{tabular}{||l||l|l|l||}
\hline \multicolumn{1}{||c||}{} & \multicolumn{3}{c||}{ Maximum Kink Energy $\left(E_{k}\right)$} \\
\hline & $\begin{array}{l}E_{k 3}=0.75 \\
\mathrm{meV}\end{array}$ & $E_{k 2}=1.5 \mathrm{meV}$ & $E_{k 1}=3.0 \mathrm{meV}$ \\
\hline \hline Max $E_{\text {diss }}(\mathrm{meV})$ & 0.0196 & 0.0392 & 0.0785 \\
\hline Avg $E_{\text {diss }}(\mathrm{meV})$ & 0.0106 & 0.0213 & 0.0425 \\
\hline Min $E_{\text {diss }}(\mathrm{meV})$ & 0.0016 & 0.0033 & 0.0066 \\
\hline Avg $E_{\text {leak }}(\mathrm{meV})$ & 0.0016 & 0.0033 & 0.0066 \\
\hline Avg $E_{\text {sw }}(\mathrm{meV})$ & 0.0090 & 0.0180 & 0.0359 \\
\hline
\end{tabular}

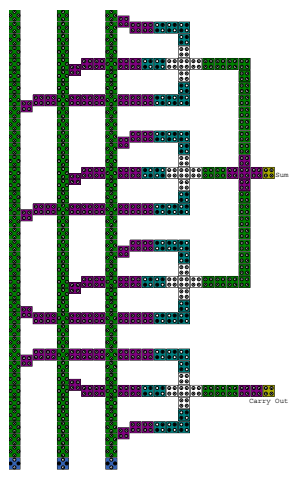

(a)

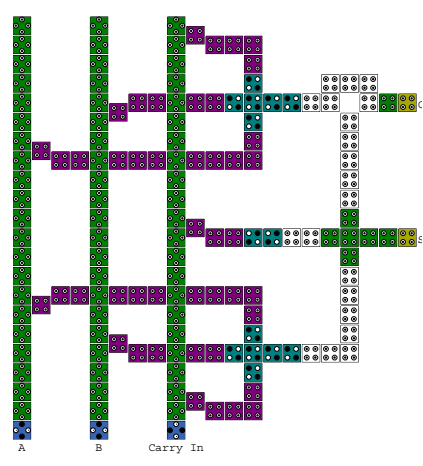

(b)
Fig. 3. Single bit QCA Adder layout(a) Adder-1 (b) Adder-2

\section{B. Switching Power}

We performed an exhaustive study on the effect of varying kink energy on the power dissipated during a switching event in a QCA circuit. In prior works, it has been shown how power dissipated in a QCA circuit varies with clock energy [11]. In this work we intend to analyze the effect of the size of a QCA cell and the kink energy associated with it on the power dissipated in the circuit.

The results of variation on power dissipation of a simple majority gate are shwon in Table IV. It can be seen that increasing the value of kink energy in a circuit leads to an increase in the overall average power dissipated in the circuit. Table $\mathrm{V}$ shows the energy dissipation in a QCA inverter for different values of kink energy.

Some very interesting observations were obtained from this study of effect of kink energy on the overall power dissipation and probability of error in QCA circuit design. We have seen that while it is desirable to design circuits with lower error probabilities (by increasing the kink energy between cells), it inadvertently increases the power dissipated in the circuit. This effect is more pronounced in larger circuits such as single bit adders (shown in Fig. 3). Table VI compares the results of output polarization at SUM node of two adders for different kink energies. As we can see that even though Adder-2 has a more efficient design and uses less number of cells, the polarization at its output is worse than that of Adder-1 for different input vector sets. Similarly, Table VII compares the energy dissipation in the two adder designs. Power dissipation in Adder-2 is greater than that of Adder1 since it has significantly more number of cells. However, we do see that the energy dissipation in a QCA circuit is 
TABLE VI

Output node Polarization AT SUM OUtPUt NODE OF ADDER-1 AND ADDER-2 QCA DESIGNS

\begin{tabular}{|c|c|c|c|c|c|c|}
\hline & \multicolumn{2}{|c|}{$E_{k 3}=1.09 \mathrm{meV}$} & \multicolumn{2}{|c|}{$E_{k 2}=2.18 \mathrm{meV}$} & \multicolumn{2}{|c|}{$E_{k 1}=4.36 \mathrm{meV}$} \\
\hline Input & Adder-1 & Adder-2 & Adder-1 & Adder-2 & Adder-1 & Adder-2 \\
\hline $\begin{array}{lll}0 & 0 & 0\end{array}$ & 0.9110 & 0.8095 & 0.9998 & 0.9964 & 1.0000 & 1.0000 \\
\hline $\begin{array}{lll}0 & 0 & 1\end{array}$ & 0.7311 & 0.8058 & 0.9935 & 0.9965 & 1.0000 & 1.0000 \\
\hline $0 \begin{array}{lll}0 & 1 & 0\end{array}$ & 0.7440 & 0.6833 & 0.9944 & 0.9667 & 1.0000 & 0.9991 \\
\hline $\begin{array}{llll}0 & 1 & 1\end{array}$ & 0.7090 & 0.6312 & 0.9931 & 0.9569 & 1.0000 & 0.9989 \\
\hline 100 & 0.7090 & 0.6312 & 0.9931 & 0.9569 & 1.0000 & 0.9989 \\
\hline 101 & 0.7440 & 0.6833 & 0.9944 & 0.9667 & 1.0000 & 0.9991 \\
\hline 110 & 0.7311 & 0.8058 & 0.9935 & 0.9965 & 1.0000 & 1.0000 \\
\hline 1111 & 0.9110 & 0.8095 & 0.9998 & 0.9964 & 1.0000 & 1.0000 \\
\hline
\end{tabular}

TABLE VII

Non-Adiabatic EnERgy Dissipation In ADDER-1 AND AdDER-2 QCA DESIGNS

\begin{tabular}{|c|c|c|c|c|c|c|}
\hline & \multicolumn{2}{|c|}{$E_{k 1}=4.36 \mathrm{meV}$} & \multicolumn{2}{|c|}{$E_{k 2}=2.18 \mathrm{meV}$} & \multicolumn{2}{|c|}{$E_{k 3}=1.09 \mathrm{meV}$} \\
\hline & Adder-1 & Adder-2 & Adder-1 & Adder-2 & Adder-1 & Adder-2 \\
\hline $\operatorname{Max} E_{\text {diss }}$ (in meV) & 3.0939 & 1.3556 & 1.5404 & 0.6778 & 0.8127 & 0.3389 \\
\hline $\operatorname{Avg} E_{\text {diss }}($ in $\mathrm{meV}$ ) & 1.7398 & 0.7650 & 0.8665 & 0.3825 & 0.4556 & 0.1912 \\
\hline Min $E_{\text {diss }}($ in $\mathrm{meV})$ & 0.4083 & 0.1949 & 0.2038 & 0.0974 & 0.1041 & 0.0487 \\
\hline $\operatorname{Avg} E_{\text {leak }}($ in $\mathrm{meV})$ & 0.4089 & 0.1956 & 0.2041 & 0.0978 & 0.1043 & 0.0489 \\
\hline $\operatorname{Avg} E_{s w}($ in $\mathrm{meV})$ & 1.3309 & 0.5693 & 0.6624 & 0.2847 & 0.3513 & 0.1423 \\
\hline
\end{tabular}

almost linearly proportational to the maximum kink energy of the circuit.

It can be seen from the results that the output node polarization error improves while power dissipation deteriorates when the kink energy is increased. Hence designers need to choose the size of QCA cells based on circuit requirements to optimize power and error. This is different from thermal studies performed on QCA circuits which resulted in increase in output error and power dissipation at higher temperatures. From the results obtained for polarization and power dissipation in small and big QCA circuits, we have clearly seen that kink energy is an important factor to design most optimum circuits at a given temperature and clock energy. Hence designers need to make careful use of kink energy as parameter for designing QCA circuits to optimize error and power.

\section{CONCLUSION}

In this work we performed error-power tradeoff studies for QCA circuits. We can see from the results that output node polarization error improves while power dissipation deteriorates when the kink energy is increased. Hence designers need to choose the size of QCA cells based on circuit requirements to optimize power and error. This is different from thermal studies performed on QCA circuits which resulted in increase in output error and power dissipation at higher temperatures. From the results obtained for polarization and power dissipation in small and big QCA circuits, we have clearly seen that kink energy is an important factor to design most optimum circuits at a given temperature and clock energy. Hence designers need to make careful use of kink energy as a parameter for designing QCA circuits to optimize error and power.

\section{REFERENCES}

[1] C. Lent and P. Tougaw, "A Device Architecture for Computing with Quantum dots," in Proceeding of the IEEE, vol. 85-4, pp. 541-557, April 1997.
[2] R. P. Cowburn and M. E.Welland, "Room temperature magnetic quantum cellular automata," Science, vol. 287, Feb 2000.

[3] M. Parish and M. Forshaw, "Magnetic Cellular Automata (MCA) systems," IEEE Proceedings of Circuits, Designs and Systems, vol. 151, pp. 480-485, 2004.

[4] C. Lent and B. Isaken, "Clocked Molecular Quantum-Dot Cellular Automata," IEEE Transactions on Electron Devices, vol. 50, pp. 18901896, September 2003.

[5] Y. Lu, M. Liu, and C. Lent, "Molecular quantum-dot cellular automata: From molecular structure to circuit dynamics," Journal of Applied Physics, vol. 102, no. 3, p. 034311, 2007.

[6] M. Choi, M. Choi, Z. Patitz, and N. Park, "Efficient and Robust Delay-Insensitive QCA (Quantum-Dot Cellular Automata) Design ," IEEE International Symposium on Defect and Fault-Tolerance in VLSI Systems, pp. 80-88, 2006.

[7] M. Tahoori, M. Momenzadeh, J. Huang, and F. Lombardi, "Defects amnd Faults in Quantum Cellular Automata at Nano Scale," in Proceedings of the 22nd IEEE VLSI Test Symposium, pp. 291-296, April 2004.

[8] M. Momenzadeh, M. Ottavi, and F. Lombardi, "Modeling QCA Defects at Molecular-level in Combinational Circuits," International Symposium on Defects and Fault Tolerance in VLSI Systems, pp. 208-216, 2005.

[9] S. Bhanja and S. Srivastava, "A Bayesian Computing Model for QCAs," NSTI Nanotechnology Conference, 2005.

[10] S. Bhanja and S. Sarkar, "Probabilistic Modeling of QCA Circuits Using Bayesian Networks," IEEE Conference on Nanotechnology, pp. 383386, June 2006.

[11] S. Srivastava, S. Sarkar, and S. Bhanja, "Power Dissipation Bounds and Models for Quantum-dot Cellular Automata Circuits," IEEE Conference on Nanotechnology, vol. 1, pp. 375-378, June 2006.

[12] P. Douglas Tougaw and C. S. Lent, "Dynamic behavior of Quantum Cellular Automata," Journal of Applied Physics, vol. 80, pp. 4722-4736, Oct 1996.

[13] J. Timler and C. Lent, "Power gain and dissipation in quantum-dot cellular automata," Journal of Applied Physics, vol. 91, pp. 823-831, January 2002.

[14] K. Walus, T. Dysart, G. Jullien, and R. Budiman, "QCADesigner: A Rapid Design and Simulation Tool for Quantum-Dot Cellular Automata," IEEE Trans. on Nanotechnology, vol. 3, pp. 26-29, March 2004.

[15] S. Srivastava and S. Bhanja, "Hierarchical Probabilistic Macromodeling for QCA Circuits," IEEE Transactions on Computers, vol. 56, pp. 174190, Feb 2007. 